\title{
Novel Approach to Develop Behavioral Model Of 12-Pulse Converter
}

\author{
Amit Sanglikar, and Vinod John, Member, IEEE
}

\begin{abstract}
A novel approach to develop behavioral model of 12pulse converter, which reduces the overall system simulation time, is presented. A detailed model of a 12-pulse converter with its control using circuit simulation tools such as PSPICE, MATLAB or SABER takes significant computer time for simulation. This is even more critical when the power converter is only a small part of a large system under study. A model based on mathematical equations derived under ideal conditions could be used, but the need to consider secondary effects such as continuous and discontinuous current or current commutation make this method much complicated due to the multiple modes of power converter operation. A regression based behavioral model was developed using a "Design of Experiment" (DOE) approach with very good statistical fit. This reduces simulation time drastically capturing also those aspects of the 12-pulse converter that are not normally included in models using other simplifications. This method could also be extended to the modeling of other complex power converter topologies and subsystems allowing a much faster simulation of full installations.
\end{abstract}

Index Terms-- Simulation, power converter model, positive sequence solution, table lookup, design of experiments.

\section{INTRODUCTION}

$\mathrm{W}$ ITH increasing electrical power demands, and loading limitations of existing power distribution networks the trend is towards distributed energy generation. Increasing use of computerized information systems demand higher power quality from the grid. The need exists for detailed system level analysis using computer simulation of power generating units such as micro-turbine, fuel cell, and power quality improvement systems such as high power UPS, Dynamic voltage restorer (DVR) and distribution Statcoms interconnected with the grid or in islanded operation. This leads us to the development of models of various power system components that may be interconnected in complex manner. The power converter can be a relatively small component of the overall system. All node voltages and branch currents of the power converter may not be necessary for the study of the overall systems. One such power electronic

Amit Sanglikar is with Tektronix Engg. Development India Pvt. Ltd, Bangalore 560001, Karnataka, India, (e-mail: amit.sanglikar@tek.com).

Vinod John is with the Department of Electrical Engineering, Indian Institute of Science, Bangalore 560012, Karnataka, India. (e-mail: vjohn@ee.iisc.ernet.in).

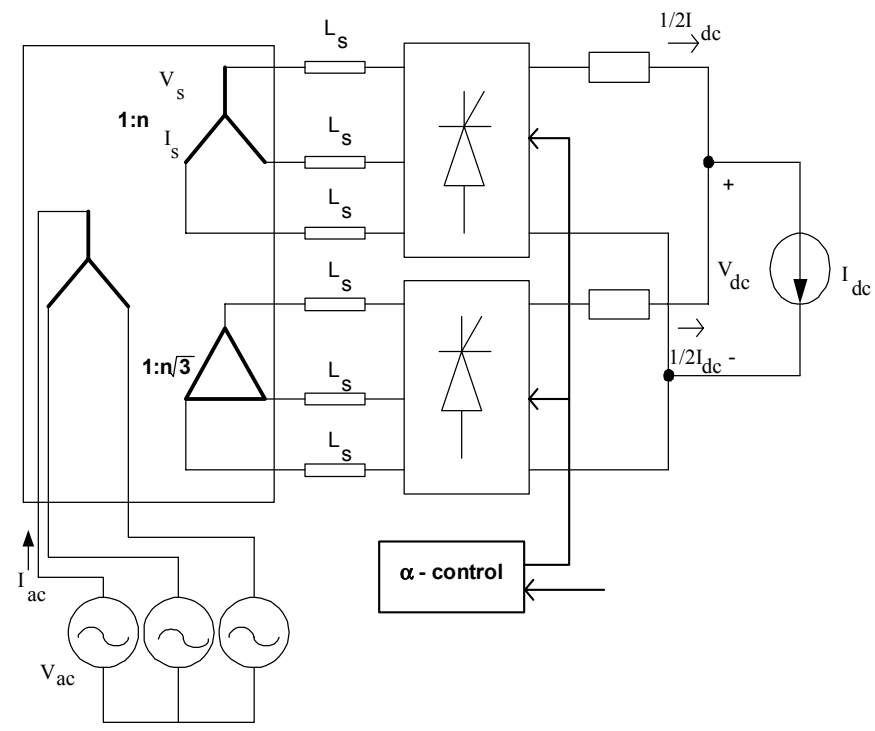

Fig. 1. Twelve-pulse converter used as rectifier front end for the UPS with the inverter side modeled as a current source load.

component is a large UPS, within which the 12-pulse converter is significant part of power electronics. For powersystem level study a simplified representation of the 12-pulse rectifier is required [1]. A prime requirement is to make use of component models of the subsystems to perform analysis such as sizing, energy storage requirements overall power quality compatibility with loads.

System level analysis of distributed power generating units using circuit simulators involves system level modeling of various sub-systems, such as 12-pulse converter in a Megawatts UPS. An analytical model defined using mathematical equations in high level simulation languages takes less simulation time. Mathematical model developed with simplified assumption limits the scope of study because secondary effects such as continuous and discontinuous current, current overlap in the thyristors during commutation etc., can not be easily analyzed since mathematical model are derived under ideal conditions [2]-[4]. A detailed component level model of 12-pulse converter can be used to take into account these secondary effects [5]. However such component level model even by itself would take significant time for simulation. Hence, there is a need for developing a model that takes into account secondary effects and can be expressed in mathematical form in order to reduce simulation time. In this paper a regression based behavioral model of a 12-pulse 

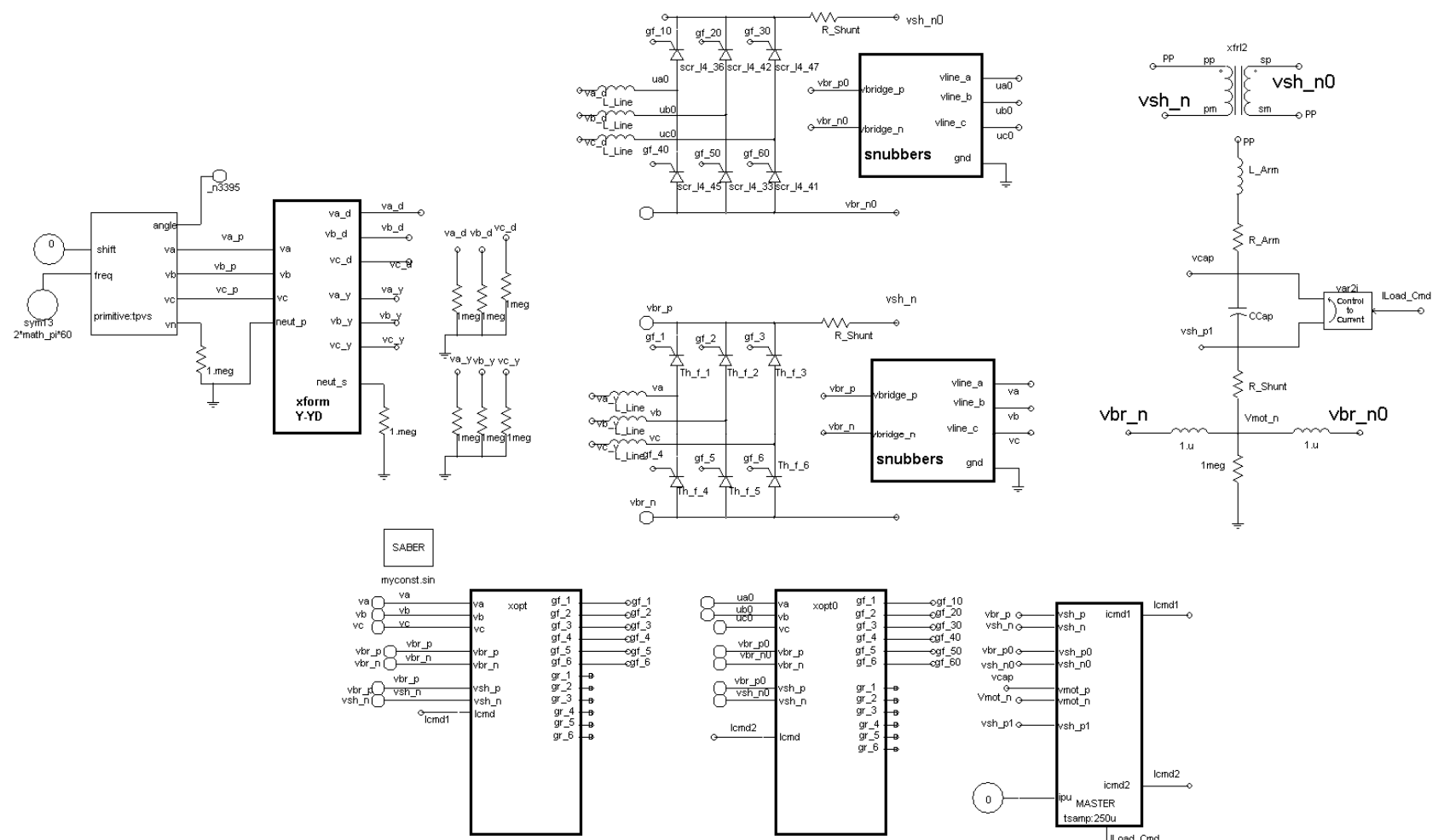

Fig. 2. Network component level circuit simulation model of a 12-pulse converter.

converter is described. The performance of the model is compared with simplified analytical models and detailed network models in terms of simulation speed and accuracy.

\section{Modeling Approach}

Modeling of the power converter is based on a three step approach consisting of the following. First is a preliminary mathematical idealized model that is used for simplified understanding. The second step is to create a detailed component level model that captures all the required nonidealities. The third step is to obtain the regression model.

\section{A. Mathematical approach}

A simplified analysis of the 12-pulse converter shown in Fig. 1 leads to the following equations for the input and output quantities.

$$
\begin{aligned}
& V_{d c}=\frac{3 \sqrt{2}}{\pi} V_{s} \cos \alpha-\frac{3 \omega L_{s}}{\pi} \frac{1}{2} I_{d c} \\
& \cos (\alpha+u)=\cos \alpha-\frac{\omega L_{s}}{\sqrt{2} V_{L L}} I_{d c} \\
& \mathrm{DPF} \approx \cos (\alpha+\mathrm{u}) \\
& V_{s} I_{s 1} D P F=V_{d c} I_{d c} / 2 \\
& I_{s 1}=\frac{V_{d c} I_{d c}}{2 V_{s} D P F}
\end{aligned}
$$

Where, $\alpha$ : firing angle

$\mathrm{u}$ : commutation interval

$I_{s 1}$ : fundamental-frequency current of $I_{s}$ n: effective transformer ratio (primary Wye and secondary Wye is $1: n)$

These mathematical relations are based on following assumptions:

- Continuous conduction of DC bus current

- Sinusoidal, balanced and constant AC voltage

- Balanced AC side impedance

- Ideal circuit component.

A model based on mathematical equations derived under ideal conditions could be used for the system level study. However the need to consider secondary effects such as continuous and discontinuous current, and different modes of current overlap in the thyristors during commutation make this method of analysis complex [6]. Deriving the equations for quantities such as Total harmonic distortion (THD) requires complex expressions along with simplifying assumptions on the interactions between the input and output filters.

\section{B. Component level modeling}

A detailed component level model of 12-pulse converter that takes into account secondary effects such as continuous and discontinuous current, current overlap in the thyristors during commutation etc. can be used. A detailed network component model of a 12-pulse converter implemented in SABER is shown in Fig. 2.

For the system level study that we are found that this model is not as useful for following reasons. One is that, this model alone already takes a long time to complete a simulation run in a computer simulation. It is not desirable for such a small component of a larger system to incur such a long model computation time. Also, very few variables of this model such 
as DC bus voltage $\left(\mathrm{V}_{\mathrm{dc}}\right)$, input current, power factor, real power, reactive power, THD etc., are the main variables required from the overall system simulation and analysis.

Simulation using the component based model allows one to capture the operation of the power converter. The primary regression functions of the operation of the 12-pulse converter was derived in terms of the firing angle and the equivalent DC bus current.

\section{Regression based model}

Regression methods can be used to develop simple empirical mathematical relationships between inputs and output variables. Regression provides an approximate means of obtaining the relationships between a response parameter and the factors that influence it when the true relationship is unknown, or is too difficult to derive. Regression involves mainly analyzing factors that affects response parameters and building equations that can be used to predict system behavior at interpolated operating points and conditions. All the input and output variables for the regression are evaluated at steady state operating conditions. Energy storage elements such as capacitors and inductors are not included in the regression equation. Hence after accounting for energy loss, the power flow between the input and the output in the circuit is balanced on an instantaneous basis in the regression model.

The first step to obtain the regression model is to identify the input (independent) variables and the output (dependent) variables. The complexity of the regression equation can rapidly increase as the number of input variables is increased. Hence, a minimum number of input variables should be used. The next step is to determine the operating range of each of the independent variables. Care should be taken to ensure that the results of the study make use of input variables within this range. Choosing a narrower operating range can help improve the accuracy of the model. The third step is to evaluate the granularity of the operating conditions under which the regression is evaluated. In case a large nonlinearity is expected in certain variables, then the number of evaluation of the system should be increased for those variables. Once these decisions are made, the simulations to obtain the regression equations is carried out using the detailed network model of 12-pulse converter a different points in the space and a nonlinear multi-variable function that can be represented as a response surface was obtained. From these simulation results, the relations between system level parameters of the 12-pulse converter and the variables on which they depend are obtained as a polynomial function of the independent variables.

\section{REGRESSION BASED BEHAVIORAL MODEL OF 12-PULSE CONVERTER}

Response parameters are system level variables for which empirical mathematical relation need to be obtained. In this case, DC bus voltage $\left(\mathrm{V}_{\mathrm{dc}}\right)$, input AC current $\left(\mathrm{I}_{\mathrm{ac}}\right)$, input power factor (pf), real and reactive power at $\mathrm{AC}$ input terminals, and input current THD are identified as response parameters

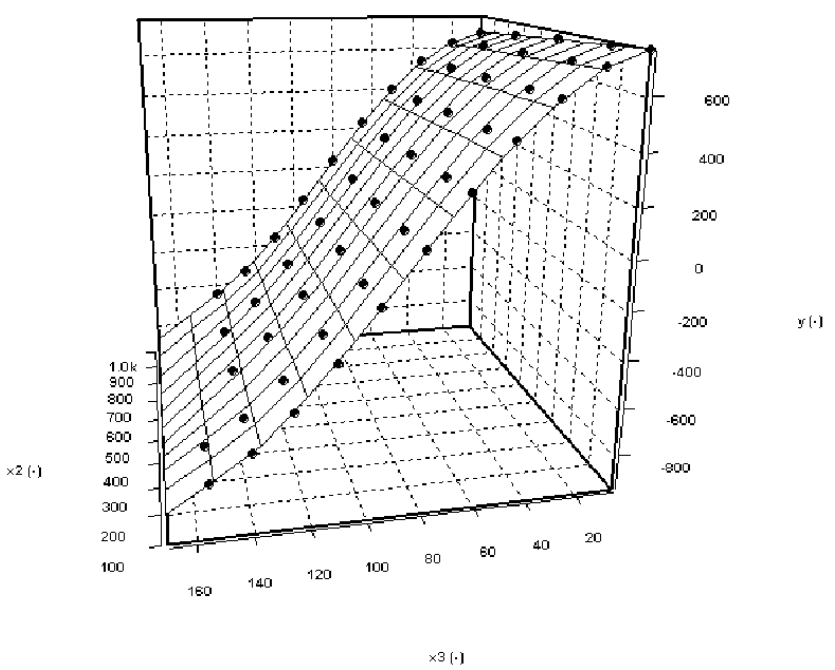

Fig. 3. Surface showing variation of DC bus voltage at different firing angle and $\mathrm{DC}$ bus current of 12-pulse converter for fixed input $\mathrm{AC}$ voltage2. $\mathrm{Y}$-axis is DC bus voltage, $\mathrm{X} 2$ axis is $\mathrm{DC}$ bus current and $\mathrm{X} 3$ axis is firing angle.

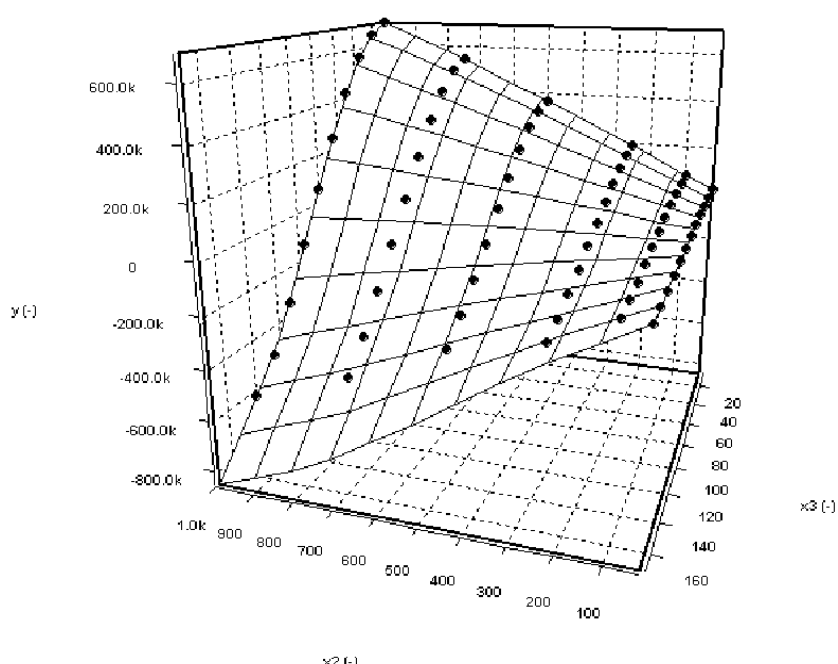

Fig. 4. Surface showing variation of real power at different firing angle and DC bus current of 12-pulse converter for fixed input AC voltage3. Y-axis is real power, $\mathrm{X} 2$ axis is $\mathrm{DC}$ bus current and $\mathrm{X} 3$ axis is firing angle.

for the system level study. Factors that influences response parameters are understood by analyzing simplified mathematical model, which are input $\mathrm{AC}$ voltage $\left(\mathrm{V}_{\mathrm{ac}}\right)$, firing angle $(\alpha)$, average DC bus current $\left(\mathrm{I}_{\mathrm{dc}}\right)$. Since regression is essentially obtaining a curve fitting equation for set of data points, various data points representing relationship between response parameters and factors that influences response parameters are required for developing regression model of 12-pulse converter. Such data points were obtained by performing design of experiment (DOE) simulations on network component level model of 12-pulse converter at different points in the design space ${ }^{1}$. A full factorial DOE with

\footnotetext{
${ }^{1}$ Design space for influencing factors are as follows: $488.75 \mathrm{~V}<\mathrm{V}_{\mathrm{ac}}<661.25 \mathrm{~V}$, $5^{0}<\alpha<175^{0}$, $25 \mathrm{~A}<\mathrm{I}_{\mathrm{dc}}<1000 \mathrm{~A}$.
} 


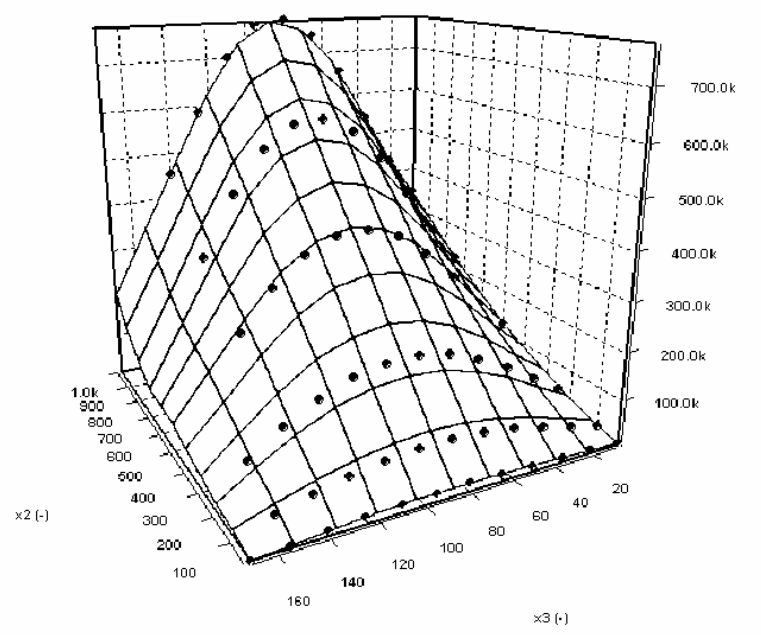

Fig. 5. Surface showing variation of reactive power at different firing angle and DC bus current of 12-pulse converter for fixed input AC voltage4. Y-axis is reactive power, $\mathrm{X} 2$ axis is $\mathrm{DC}$ bus current and $\mathrm{X} 3$ axis is firing angle.

6 levels of DC bus current, 12 levels of firing angle and 3 levels of Vac was performed to obtain data for the regression model. These data points obtained from simulation can be represented as non-linear multi-variable response surface. Response surface for some response parameters are shown in Fig. 3 through Fig. 5. Fig. 3 shows the variation of DC bus voltage with respect to firing angle indicating cosine relation, with a droop as a function of load current. Fig. 4 shows that for firing angle less that $90^{\circ}$ real power is positive and for firing angle greater that $90^{\circ}$ real power is reported as negative, as expected from the mathematical model. Fig. 5 shows reactive power is maximum when firing angle close to $90^{\circ}$ and reactive power approaching zero for firing angle of $0^{\circ}$ and $180^{\circ}$. Next step is to obtain curve fit equation; various statistical software tools such as MINITAB are available for such purpose. Curve fit equations are obtained for $V_{d c}, I_{a c}$, input power factor, real and reactive power at $\mathrm{AC}$ input terminals. A specimen third-order regression equation for DC bus voltage is shown below.

$$
\begin{aligned}
\mathrm{V}_{\mathrm{dc}} & =(\mathrm{k} 0)+\mathrm{V}_{\mathrm{ac}}(\mathrm{k} 1)+\mathrm{I}_{\mathrm{dc}}(\mathrm{k} 2)+\alpha(\mathrm{k} 3) \\
& \left.+\mathrm{V}_{\mathrm{ac}} \mathrm{d}_{\mathrm{dc}} \mathrm{k} 4\right)+\mathrm{V}_{\mathrm{ac}} \alpha(\mathrm{k} 5)+\mathrm{I}_{\mathrm{dc}} \alpha(\mathrm{k} 6)+\mathrm{I}_{\mathrm{dc}}{ }^{2}(\mathrm{k} 7) \\
& +\alpha^{2}(\mathrm{k} 8)+\mathrm{I}_{\mathrm{dc}}{ }^{2} \mathrm{~V}_{\mathrm{ac}}(\mathrm{k} 9)+\mathrm{I}_{\mathrm{dc}}{ }^{2} \alpha(\mathrm{k} 10)+\alpha^{2} \mathrm{~V}_{\mathrm{ac}}(\mathrm{k} 11) \\
& +\alpha^{2} \mathrm{I}_{\mathrm{dc}}(\mathrm{k} 12)+\mathrm{I}_{\mathrm{dc}}{ }^{3}(\mathrm{k} 13)+\alpha^{3}(\mathrm{k} 14)
\end{aligned}
$$

The regression coefficients for the above equation are in Appendix. Conducting a larger number of simulation runs allows the flexibility to incorporate higher order terms in the above equation. However, a larger number of simulation runs would require additional computation time. In the above equation some regression coefficients are not significant and hence are ignored. The statistical significance of each term in the equation provides the guidance as to whether to include the term in the equation. For example, the square term for Vac is ignored in (6). Such statistical significance can be evaluated by set confidence interval and the p-value obtained from hypothesis testing [7]. The equations are valid in the range of inputs where the detailed component simulations were carried out. Care should be taken not to extrapolate out of this range.

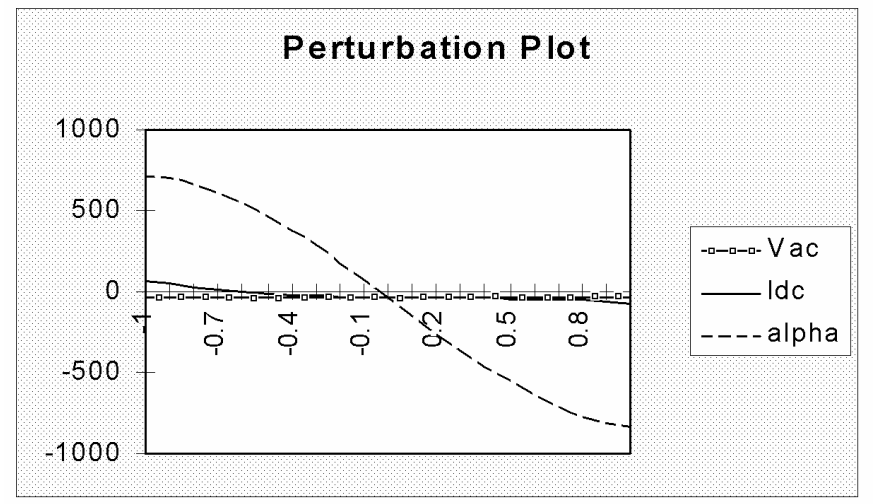

Fig. 6. Perturbation plot for DC bus voltage.

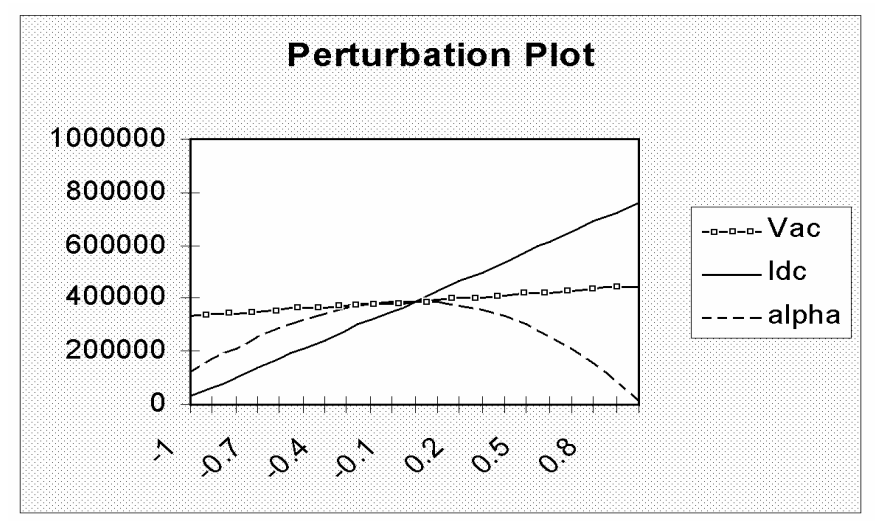

Fig. 7. Perturbation plot for reactive power.

The measure of goodness of curve fit is determined by the $\mathrm{R}^{2}$ value of the regression equation [7]. A larger number of DOE runs and use of appropriate higher order terms for the regression equations gives a better $R^{2}$ value. The $\mathrm{R}^{2}$ for most of the responses such as $\mathrm{V}_{\mathrm{dc}}, \mathrm{I}_{\mathrm{ac}}$, real and reactive power and power factor was more than $99 \%$ and THD was more than $80 \%$. The mathematical expression captures the underlying physics of the system. This can be explained by analyzing perturbation plots. Fig. 6 shows perturbation plot for DC bus voltage. The variation of DC bus voltage with respect to firing angle shows cosine relationship. Similarly Fig. 7 shows perturbation plot for reactive power. The variation of reactive power with respect to firing angle is sine variation and with input $\mathrm{AC}$ voltage is linear. This variation observed is identical to what we observe in mathematical model derived under ideal conditions.

The regression based behavioral model of 12-pulse converter modeled in SABER is shown in Fig. 8, all regression equations are defined in simple high level simulation language. The output of model is a steady state numerical value of the response parameter and not timedomain waveform. The regression model represents on the quasi steady state operation of the system and the controller that is used to model the overall system as shown in Fig. 8 does not indicate the high speed response capability of the system. The control system includes an inner dc link current controller and outer dc bus voltage controller. 


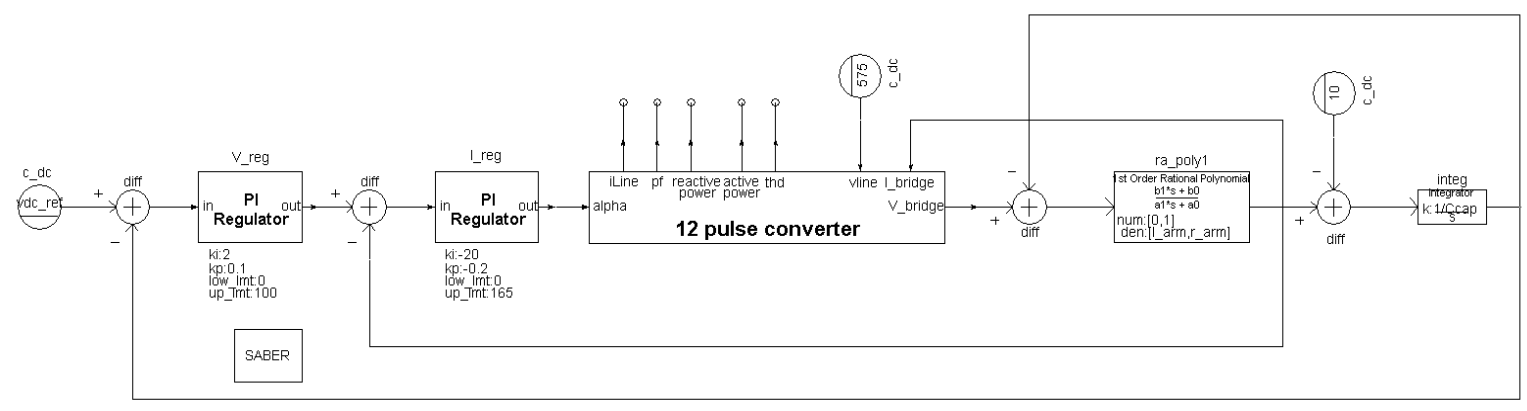

Fig. 8. Regression based behavioral model of 12-pulse converter.

Regression based model is significantly simplified and takes a small fraction of the original computational time. The computation time for the behavioral model is 0.02 seconds compared to 183 seconds for the detailed network model for 2 seconds of circuit simulation in time domain. This helps to reduce simulation time especially for system level analysis of multiple interconnected power converters. It is also possible to extend this type of regression model wherein number of distributed power units of various types is interconnected an a large number of system configurations. This model not only reduces simulation time significantly but also takes into account secondary effects such as continuous and discontinuous current, current overlap in the thyristors during commutation etc., which are would require multi-mode mathematical modeling approach.

Regression based behavioral model of 12-pulse converter developed here is not a generalized model. This model is valid only for component values (such as DC bus capacitor, filter inductor etc.) and other system parameters that are used in component level model and design space within which data points were collected. Furthermore, instantaneous timedomain waveform of response variables is not captured; the model calculates only steady-state numerical value of the response parameter, however slow control loop dynamics and slow time domain transients can be studied with the model.

A final system consisting of the grid power supply, standby genset, an UPS with a 12-pulse rectifier and inverter, and multiple flywheels for energy storage was studies with this approach. It was possible to study various operating scenarios, using this approach with significantly reduced simulation time. It was seen that the predicted operating scenarios matched well with tests conducted on a industrial power system under study.

\section{CONCLUSION}

A simplified regression based behavioral model of 12-pulse converter is presented. This model reduces simulation time significantly and also takes into account secondary effects that are difficult to model using analytical mathematical modeling approach. However, this model is not a generalized model and detailed time-domain waveforms of response variables cannot be obtained. Preliminary study indicates the usefulness of the proposed novel modeling approach for system level analysis using computer simulation.

\section{APPENDIX}

The UPS parameters considered are $\mathrm{Vs}=575 \mathrm{~V}$, frequency $=$ $60 \mathrm{~Hz}$, nominal power $=500 \mathrm{~kW}, \mathrm{Vdc}$ nominal $=540 \mathrm{~V}$.

Regression coefficients for (6) are given by: $\mathrm{k} 0=-168.22$; $\mathrm{k} 1=1.64 ; \mathrm{k} 2=9 \mathrm{e}-2 ; \mathrm{k} 3=9.26 ; \mathrm{k} 4=-7.89 \mathrm{e}-4 ; \mathrm{k} 5=-1.29 \mathrm{e}-2$; $\mathrm{k} 6=-2.92 \mathrm{e}-3 ; \mathrm{k} 7=4 \mathrm{e}-4 ; \mathrm{k} 8=-1.41 \mathrm{e}-1 ; \mathrm{k} 9=5.17 \mathrm{e}-7 ; \mathrm{k} 10=$ $1.15 \mathrm{e}-6 ; \mathrm{k} 11=-3.6 \mathrm{e}-5 ; \mathrm{k} 12=1.2 \mathrm{e}-5 ; \mathrm{k} 13=-4.21 \mathrm{e}-7 ; \mathrm{k} 14=$ $5.8 \mathrm{e}-4$.

\section{REFERENCES}

[1] Tsorng-Juu Liang, Jiann-Fuh Chen, Kuen-Jyh Chen, "Analysis of 12 pulse phase control AC-DC converter", IEEE 1999 International conference on Power electronics and Drives Systems, PEDS'99, Hong Kong, July 1999.

[2] Leland A. Schlabach, "Analysis of discontinuous current in a 12-pulse thyristor DC motor drive", IEEE Trans. on Industry Applications, Vol. 27, No. 6, Nov/Dec 1991.

[3] K. R. Padiyar, Sachidanand, A. G. Kothari, S. Bhattacharyya, A. Srivastava, "Study of HVDC controls through efficient dynamic digital simulation of converters", IEEE Trans. on Power Delivery, Vol. 4, No. 4, Oct. 1989.

[4] Keiju Matsui, Kazuo Tsuboi, Saburo Muto, Koji Iwata, "A dual thyristor converter reducing harmonics of power supply without input transformer," Conference Record of the 1991 IEEE Industry Applications Society Annual Meeting, Page(s): 925 -931 vol.1, 1991

[5] V. Rajagopalan, "Modeling and simulation of power electronic converters for power supplies", Industrial Electronics, Control, and Instrumentation, 1995, Proceedings of the 1995 IEEE IECON 21st International Conference, Page(s): 27 -32 vol. 1, 1995.

[6] T.H. Barton, Rectifiers cycloconverters and AC Controllers, Oxford Science Publications, Clarendon Press, 1994.

[7] T. Hill, P. Lewicki,. Statistics Methods and Applications, Electronic Statistics Textbook, StatSoft, Inc., Tulsa, OK, 2006. Available: http://www.statsoft.com/textbook/stathome.html. 